\title{
Méthodologie pour une simulation des transformations granulométriques de sables de la zone d'avant-côte
}

\author{
Jean-Paul BARUSSEAU ${ }^{\text {a }}$, Mohamodoul B. DIOUF ${ }^{b}$, Marc de la BARDONNIE ${ }^{c}$, Nagui EL GHANDOUR ${ }^{a}$ \\ ${ }^{a}$ Centre de formation et de recherche sur l'environnement marin, université de Perpignan, 52, av. de Villeneuve, \\ 66860 Perpignan, France \\ ${ }^{b}$ Département de géologie, université de Dakar, Sénégal \\ ${ }^{\mathrm{c}}$ Centre d'études fondamentales, université de Perpignan, France
}

(Reçu le 15 mars 1997, révisé le 31 juillet 1998, accepté le 20 août 1998)

\begin{abstract}
Methods to simulate textural evolution of mature grain-size distributions in the offshore zone. The grainsize of mature coastal sands changes rapidly in response to the variation of the energy regime which provokes either erosion or deposition of fractions of the textural assemblage. Whatever the dynamic conditions, questions of paramount importance such as the size of grain populations transported simultaneously, the most common size of the grains in movement and the grain-size range of the sediment fraction involved are still poorly understood. The present paper deals with an attempt to describe this fraction without any consideration of the processes responsible for the observed changes. A major problem results from the fact that the distribution of the mobilized fraction cannot be known and described by direct comparison between the initial and the final grain-size curves. The only way to characterize the mobile fraction between two subsequent grain-size states is to simulate various kinds of removal or deposition. The first question is to express the shape of the actual distributions of the sediments mathematically. The simple Gaussian approximation being irrelevant, distributions can be expressed using advanced computer programmes which can provide mathematical expressions for all distributions. The cubic-spline approximation was chosen. The mobile fraction was assumed to have a Gaussian distribution. Various tests were made in order to simulate qualitative phenomena observed in nature (bimodality, grain-size parameter changes...) and to assess the three parameters which define the characteristics of the fraction deposited or removed: modal value, dispersion of the mobile population and relative amplitude of the change. An application was carried out on the Senegalese coast offshore from the Senegal delta and on the Mediteranean coast in the Gulf of Lions. It suggests that the dynamic agents have a great selectivity. Further developments are considered. C Elsevier, Paris / Ifremer / Cnrs / Ird
\end{abstract}

\section{grain-size / mobile fraction / modelling / simulation}

Résumé - La connaissance des relations hydrosédimentaires dans la zone littorale et son avant-côte souffre d'une insuffisante définition du spectre complet des tailles de grains érodés, transportés et déposés sous l'action des agents dynamiques. Le problème est complexe car l'échantillonnage de la totalité du matériel mobilisé, en charriage, en saltation et en suspension, est difficile. Une solution alternative consiste à confronter les courbes granulométriques du matériel avant et après sa transformation texturale par un agent hydrodynamique. La procédure directe est toutefois invalide dans la mesure où ces courbes illustrent des distributions relatives, non comparables car non représentatives de la transformation réelle, absolue qui a eu lieu. Il est possible en revanche de faire apparaitre les effets de changements négatifs (érosion de particules) ou positifs (dépôt) pour autant que l'on puisse obtenir une formalisation mathématique de la relation taille-fréquence pour toute distribution réelle et opérer sur cette distribution des soustractions ou additions de particules selon une loi déterminée. Ces deux étapes peuvent être franchies, d'une part, en utilisant des approximations de type cubic-spline, aisément disponibles dans certains logiciels, d'autre part, en supposant que la population mobile est distribuée selon une fonction simple. On montre, à l'aide de deux exemples, l'un relatif à un départ de particules, l'autre à une arrivée de maté- 
riel se mélangeant à un sédiment antérieur en place, que cette méthode permet, en partant de la distribution initiale, de modéliser la transformation qui conduit à la distribution finale. La population mobile est ainsi totalement définie par une loi de distribution, caraclérisée par un paramètre central $\mathrm{M}$, un paramètre de dispersion $\sigma$ et par un facteur d'influence $P$, représentant l'ampleur du remaniement (positif ou négatif) dans l'intervalle dimensionnel $\mathrm{M} \pm 2 \sigma$. (C) Elsevier, Paris / Ifremer / Cnrs / Ird

\section{granulométrie / fraction mobile / modélisation / simulation}

\section{INTRODUCTION}

Il est largement admis que l'aptitude des sédiments à être remobilisés dépend de la distribution granulométrique complète des grains $[14,15,24,28,29,33,41]$. L'action des écoulements lors des processus d'érosion modifie la distribution du sédiment initial par enlèvement d'une partie plus ou moins importante des grains qui le constituent. Les conditions du transport engendrent un spectre granulométrique du matériel déplacé et déterminent la distribution du flux des particules qui se déposent, modifiant par conséquent la texture du sédiment dans lequel se mélange cet apport. Dans la pratique, cependant, on continue d'utiliser un petit nombre de données, parfois même une seule (la médiane), pour caractériser ces distributions, ce qui a pour effet de conduire à des désaccords entre les observations et les prédictions de mouvement débutant, de transport et de dépôt. Ces désaccords sont d'autant plus grands que la distribution englobe un large spectre de particules [43]. Des tentatives ont été faites pour entrer dans les modèles des représentations multi-classes du sédiment mais la durée des traitements informatiques oblige à en limiter l'application [6].

De nombreux auteurs ont cherché à analyser les changements progressifs qui marquent les différents états des sédiments au cours des phases de remaniement ou de dépôt $[18,27,44]$. Cette reconnaissance est l'objet de la démarche de McLaren [28] et de McLaren et Bowles [29] pour tenter de modeliser les transformations auxquelles est soumis un sédiment défini par un ensemble de classes granulométriques. Ils ont supposé que les petits grains ont une probabilité de transport plus élevée que les grains de taille plus grande. Le phénomène peut être schématisé comme une suite d'extractions et d'apports qui tendent à transposer la forme de la distribution initiale vers celle de la distribution terminale par l'intermédiaire d'un flux exporté et/ou importé.

Il existe une grande incertitude sur la forme réelle de la distribution de ce matériel, lien entre deux états succes- sifs du sédiment. La sélection des grains par les mécanismes de transport conduit à la construction de distributions sédimentaires considérées par les uns comme gaussiennes $[11,48]$ ou comme des mélanges de populations normales [32]. D'autres, avec McLaren et Bowles [29], rappellent que le milieu empêche une parfaite adéquation de la distribution normale avec les distributions réelles en raison de la complexité des processus d'entraînement et de dépôt et de l'influence du matériel originel. Ce point de vue est justifié aussi par le fait que l'analyse et la compréhension des différences existantes sont rendues difficiles en raison du manque de connaissances qui s'observe, notamment dans le domaine des relations hydrosédimentaires [7, 35].

Le but du présent travail est de rendre compte de la modification texturale accompagnant les évolutions granulométriques réelles observées dans un environnement marin. Pour cela, il convient: (1) de trouver une expression des courbes granulométriques réelles permettant l'interpolation de la valeur de fréquence associée à toute dimension de l'intervalle granulométrique considéré, (2) d'effectuer sur les distributions initiales ainsi formalisées des extractions ou des adjonctions de particules réparties, en première étape, sclon des lois simples, (3) de vérifier, en utilisant une démarche d'essais et d'erreurs, que les paramètres des fractions mobiles testées conduisent bien à l'obtention des distributions terminales réellement observées sur le terrain.

Les conditions naturelles qui régissent les redistributions de grains [25] ou les modalités de triage [36] ne sont pas considérées ici. En effet, la connaissance des processus physiques et des phénomènes hydrosédimentaires (mise en mouvement, transport, sélection dimensionnelle des grains au cours du déplacement) demeureront très spéculatifs précisément tant que la distribution granulométrique des fractions mobilisées et sa relation avec la répartition de fréquence des tailles dans le matérielsource ne seront pas élucidées. 


\section{MÉTHODE}

\subsection{Principe}

Dans ce qui suit, on a supposé qu'à toute particule est associée une probabilité de mobilisation ou, inversement, de mise en place, dès lors que le critère de seuil [26] est dépassé. Cette probabilité s'exprime par la distribution des tailles de grains extraits ou apportés formant une fraction mobile.

La mise en évidence de cette distribution n'est toutefois pas aisée. Comme elle représente la différence entre la répartition de fréquence des tailles de grains avant et après le phénomène responsable de la modification granulométrique, une première solution pourrait être de soustraire la courbe de fréquence granulométrique « finale » de la courbe « source » observée. Elle conduit à une impasse dans la mesure où, les représentations granulométriques étant relatives, les différences observées ne sont, en fait, que des artefacts. Cette difficulté, qui n'a pas toujours été évitée $[42,47]$, est bien soulignée par McLaren et Bowles [29].

La seule solution pratique consiste par conséquent à simuler des enlèvements ou des arrivées de particules selon une loi de fréquence déterminée à partir d'une distribution originale connue et de recalculer la courbe conséquente de façon à aboutir à une répartition qui coïncide, aussi précisément que possible, avec la distribution observée après la phase de modification de la texture. Pour cela, il convient de définir la loi de transformation granulométrique. McLaren et Bowles [29] considèrent que la fonction de distribution des particules mobilisées augmente de façon monotone en échelle phi. Nous n'avons pas repris cette idée car des expériences variées [3] tendent à montrer que le matériel en transport présente une distribution à maximum, voisine d'une distribution de Gauss. La masse de particules extraites se réduit de part et d'autre d'un mode granulométrique caractéristique du phénomène d'érosion. On a donc admis, à la suite également des observations de Sengupta [39], que les populations mises en mouvement sont réparties selon une loi de Gauss.

Dans chacun des tests réalisés, la distribution de la fraction mobile (en érosion ou en apport) a été définie par trois valeurs : (1) le mode $M$ de la population de grains extraite (fraction mobile), (2) son écart type $\sigma$ et (3) l'ampleur $\mathrm{P}$ de la transformation, c'est-à-dire le pourcentage de matériel enlevé ou ajouté par rapport au matériel initial dans un intervalle de $\pm 2 \sigma$ autour de $\mathrm{M}$. La pre- mière série de tests, purement formelle, illustre la méthode, de façon à simplifier la compréhension de la démarche et à valider qualitativement les changements obtenus.

Plusieurs séries de tests sont ensuite présentées en tenant compte de situations réelles observées dans le cas d'avant-côtes microtidales à forte dominance des houles.

\subsection{Provenance des échantillons}

Les échantillons étudiés proviennent de deux secteurs d'avant-côte. Les prélèvements ont été réalisés sur les mêmes points de profils répétés dans le temps. La localisation précise des points a été contrôlée à l'aide d'un théodolite-tachéomètre. L'appareil était positionné sur un repère permanent et les profils définis en fonction d'une référence angulaire connue à $\pm 10^{-4}$ grade près. Les distances sur le profil étaient mesurées à $\pm 1 \mathrm{~m}$ près. Les prélèvements ont été réalisés en plongée en même temps que des observations du fond qui ont montré l'homogénéité du voile sédimentaire aux profondeurs où ils ont été collectés $(5-10 \mathrm{~m})$. À ces profondeurs, on a estimé que les remaniements physiques et la bioturbation étaient suffisamment modérés dans les conditions locales pour que seule la pellicule de surface du sédiment soit affectée par les changements texturaux. Le matériel récolté représente donc le niveau superficiel $(0-1 \mathrm{~cm})$.

Un premier secteur se situe sur l'avant-côte de la Langue de Barbarie (Sénégal), étudiée à l'occasion d'un programme de recherche morphodynamique. Au cours des 4 années d'observation (1988-1991), les prélèvements ont montré des degrés variés de variabilité tant spatiale que temporelle. Les variations spatiales sont très fortes dans le haut des profils étudiés, notamment au niveau d'un épaulement passant parfois à une barre, l'un et l'autre situés vers $3 \mathrm{~m}$ de profondeur; en revanche, elle est moindre et plus progressive aux profondeurs inférieures à $5 \mathrm{~m}$ [30]. Dans tous les cas, la variabilité granulométrique temporelle est sensible quand on considère les sédiments avant et après la période des fortes houles estivales, également responsables d'une érosion des fonds sur l'ensemble de l'avant-côte. Cette évolution est conforme aux observations habituelles puisque les changements morphodynamiques les plus importants sur les côtes sableuses surviennent à l'occasion des événements les plus énergiques [17]. Les échantillons étudiés ici proviennent d'un point situé à $10 \mathrm{~m}$ de profondeur sur un des profils. Ce matériel peut être considéré comme représentatif des sédiments de cette profondeur et les change- 
ments observés au cours du temps ne reflètent donc que l'incidence de la variation temporelle pré- et post-tempêtes d'hivernage.

Le second secteur est situé sur l'avant-côte du golfe du Lion à Sète. Les échantillons furent recueillis sur un profil rigoureusement positionné, à une profondeur de $5 \mathrm{~m}$, avant et après un coup de mer (19-20 octobre 1994) qui avait provoqué une légère diminution de profondeur (accumulation) au large des barres d'avant-côte [1]. Cet auteur a montré que les variations granulométriques sont très faibles dans l'espace au-delà du système des barres d'avant-côte qui caractérisent le golfe du Lion. Sur le versant qui les précède vers le large (glacis d'avant-côte), les transitions granulométriques sont extrêmement progressives, traduisant seulement l'atténuation de la houle avec la profondeur [19]. Les échantillons étudiés sont donc représentatifs de leur domaine de profondeur. En outre, la précision du positionnement du point de prélèvement garantit également la validité de la comparaison des états successifs du sédiment en ce point.

Les caractères morphologiques et hydrodynamiques des deux secteurs ne sont pas présentés dans ce qui suit en raison de l'objectif méthodologique affiché.

Les distributions granulométriques ont été obtenues par tamisage des échantillons lavés, sans plus ample traitement. Les sédiments étudiés sont principalement formés de grains de quartz équidimensionnels. Les teneurs en éléments bioclastiques sont généralement faibles $(<15 \%)$. En outre, dans la mesure où les matériaux récoltés sont fins dans les deux cas, on a considéré que les écarts de forme des éléments coquilliers par rapport aux grains quartzeux subsphériques, avaient peu d'importance dans le comportement hydrosédimentaire de l'ensemble des particules. Les valeurs corrigées des mailles de tamis ont été obtenues au microscope ou par tamisage d'un échantillon standard. Les dimensions sont indiquées en échelle des phi, seule façon de donner un sens à la notion d'écart type.

\section{RÉSULTATS}

\subsection{Cas théorique}

Le premier test réalisé représente un enlèvement de grains (extraction) à partir d'une distribution initiale hypothétique que, par simplification, on a supposé respecter la loi normale. On a fait varier les trois paramètres directeurs (mode granulométrique $\mathrm{M}$, déviation standard $\sigma$ et quantité extraite $P$ ) de la façon indiquée dans le tableau $l$.

Tableau I. Paramètres de la distribution d'origine et mode granulométrique $(M)$, écart type $(\sigma)$ et pourcentage $(P)$ caractérisant la population enlevée dans le test de la figure 1 .

Table I. Grain-size mode (M), standard deviation $(\sigma)$ and quantity modified (P) for the first test.

\begin{tabular}{lcccc}
\hline & $\begin{array}{c}\mathbf{M} \\
\text { (unités } \phi)\end{array}$ & $\begin{array}{c}\mathbf{M} \\
(\mathbf{m m})\end{array}$ & $\begin{array}{c}\sigma \\
\text { (unités } \phi)\end{array}$ & $\begin{array}{c}\mathbf{P} \\
(\%)\end{array}$ \\
\hline Population initiale & 1,376 & 0,38 & 0,713 & - \\
Population enlevée & 1,75 & 0,3 & 0,25 & 10 \\
& & & 0,50 & 30 \\
& & & 0,75 & 50 \\
\hline
\end{tabular}

Les résultats ont été obtenus en utilisant le logiciel Math$\mathrm{Cad}^{\circledR}$ et en appliquant une condition de limitation de l'extraction dès lors que l'élimination théorique dépassait les disponibilités en matériel dans la population initiale.

La figure 1 montre, sur la première rangée de diagrammes, la forme de la population initiale et les extractions opérées en fonction des paramètres du tableau $I$; sur la deuxième rangée, le résultat des extractions réalisées en fonction d'écarts types progressivement plus larges (colonne A: $\sigma=0,25 \phi$, colonne B: $\sigma=0,50 \phi$ et colonne $C:(\sigma=0,75 \phi)$ et sur la troisième rangée, la même information mais sous forme cumulative.

On observe que la simulation reproduit correctement des phénomènes couramment observés dans la nature quand la distribution est modifiée sous l'effet des agents naturels du milieu. Des bimodalités surgissent lorsque le prélèvement lognormal est important ( 30 à $50 \%$ ) et que son écart type est faible (colonne A de la figure 1). Des « raideurs » dans le tracé des queues de distribution peuvent, comme on le constate fréquemment dans les milieux naturels, cacher des bimodalités naissantes (figure $\mathrm{I}$, col. B, courbe correspondant à une extraction $\mathrm{P}=50 \%$ ). Les indices de position et de triage sont modifiés quand la dispersion de la fraction extraite est grande $(\sigma=0,5$ et $0,75 \phi)$. On voit ici que les différents paramètrcs granulométriques, qu'ils mesurent le triage général du sédiment $(\sigma)$ ou des triages relatifs entre les différentes fractions constitutives de la distribution (asymétrie, angulosité), dépendent non seulement de la composition dimensionnelle du sédiment initial mais aussi des carac- 

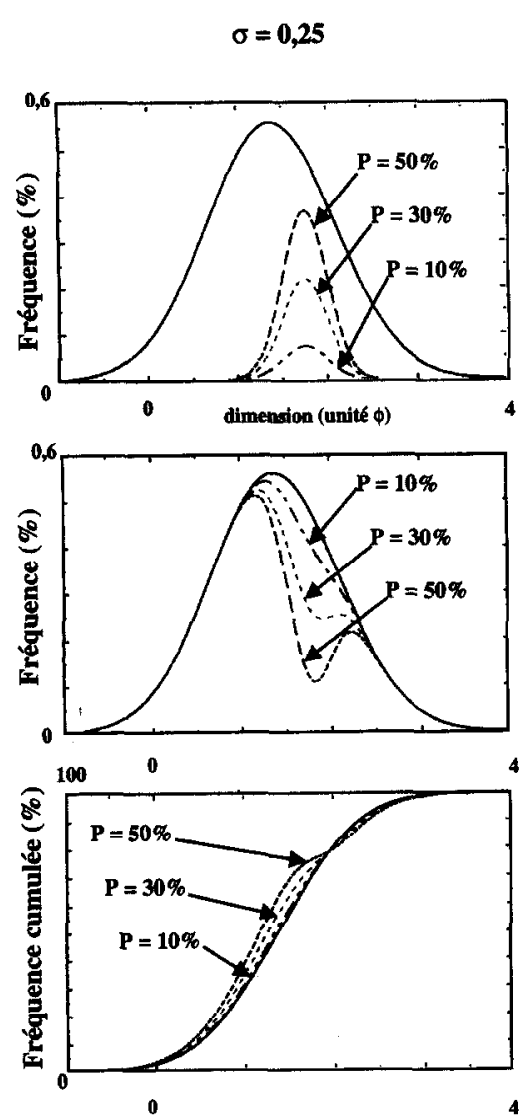

A
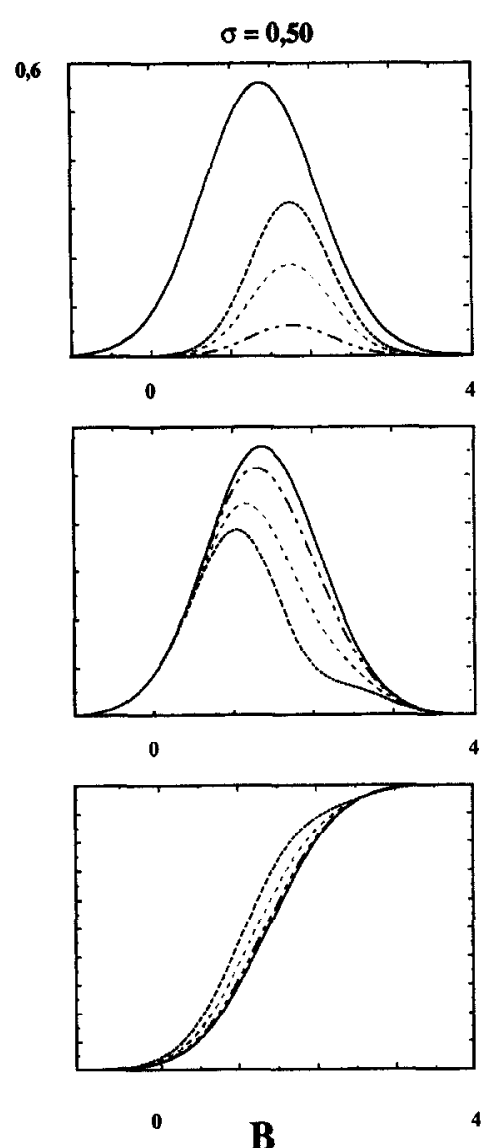

$\sigma=\mathbf{0 , 7 5}$

0,6
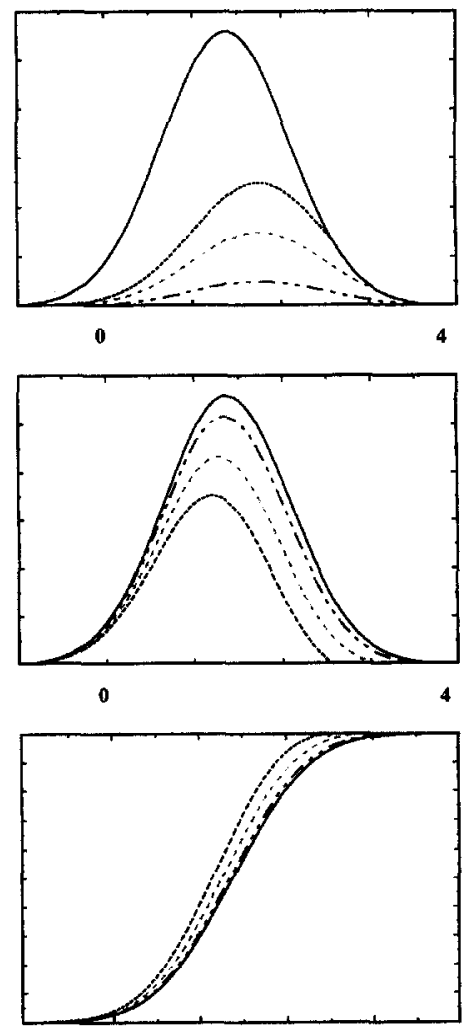

$\mathbf{C}$
4

Figure 1. Simulation d'enlèvement de particules normalement distribuées à partir d'une distribution théorique. Influence de l'êcart type de la distribution extraite.

Chaque colonne se rapporte à une distribution d'écart type défini $(\sigma=0,25 \Phi$ pour les trois graphes de la colonne $A, \sigma=0,5 \Phi$ pour la colonne $\mathrm{B}, \sigma=\mathbf{0 , 7 5 \Phi}$ pour la colonne $\mathrm{C}$ ). La première ligne de graphiques indique, d'une part, la distribution théorique initiale (en trait gras), supposée gaussienne pour simplifier, et d'autre part, les distributions, également gaussiennes, des populations extraites (en tirets), chacune centrée sur une valeur modale commune $(\mathrm{M}=1,75 \phi ; 0,296 \mathrm{~mm})$ mais avec différents cas représentant l'ampleur de l'extraction (P en $\% ; 10,20,50$ étant le pourcentage du matériel disponible enlevé dans l'intervalle $\mathrm{M} \pm 2 \sigma$ ). La deuxième rangée (même légende) indique le résultat de l'extraction comparé à la distribution initiale. La troisième rangée présente le même résultat sous forme cumulative semi-logarithmique.

Figure 1. Simulation of extractions from theoretical distributions.

Each column is devoted to a particular standard deviation (top: column A: $\sigma=0.25 \Phi$, column B: $\sigma=0.5 \Phi$, column C: $\sigma=0.75 \Phi$ ). The first row of sketches gives the initial Gaussian distribution (heavy line) and the distributions of extracted fractions (dotted lines) based on a Gauss function with the same modal value $(M=1.75 \phi ; 0.296 \mathrm{~mm})$ and various quantities extracted $(\mathrm{P}$ in \%). The second row (same legend) gives the results of the extractions in each case. The third row provides the same data but reshaped in the relative format of a log-cumulative graph.

téristiques des populations granulométriques apportées et mélangées («brassage vertical » [3]) à l'assemblage initial ou extraites de celui-ci. Les paramètres apparaissent ainsi moins comme de simples descripteurs quantitatifs de la forme de la distribution que comme des témoins d'un héritage entre la structure granulométrique du sédiment initial d'une part et celle du stock mobile ajouté ou retranché d'autre part. On voit également que les indices de skewness et kurtosis sont également modifiés (figure 1 , col. C). 
J.-P. BARUSSEAU et al.

'Tableau II. Granulométrie d'un matériel sédimentaire réel et paramètres des populatious soustraites.

Table II. Grain-size results for a marine sand and parameters used for the tested extractions

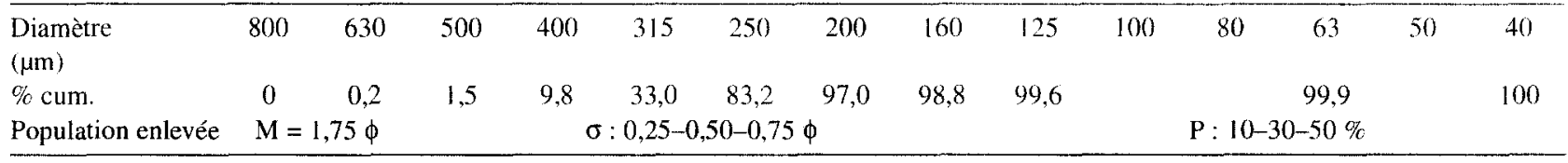

\subsection{Résultats sur des sédiments réels}

\subsubsection{Formalisation de la courbe granulométrique réelle}

Le traitement numérique à partir d'une granulométrie réelle implique qu'une loi de distribution lui soit affectée pour tout l'intervalle dimensionnel représenté. I'application d'une distribution de Gauss ayant les mêmes caractères de paramètre central et de triage n'est pas satisfaisante [21]. On a préféré utiliser des interpolations de type cubic-spline qui permettent une formalisation mathématique plus exacte. L'interpolation cubic-spline est largement utilisée pour ajuster une fonction à une série de points expérimentaux de telle façon que les dérivées première et seconde de la courbe ajustée soient continues. La courbe expérimentale est alors transformée en une série de courbes adjacentes et partiellement chevauchantes répondant chacune à une équation cubique dans laquelle chaque point est connecté au suivant. Différentes hypothèses peuvent être adoptées pour la terminaison aux extrémités : linéaire (Ispline), de second degré (pspline) ou courbe cubique (cspline). La représentation mathématique correcte d'une granulométrie réelle connue d'après les données expérimentales (tableau $I I$ ) étant ainsi assurée, le calcul automatique des extractions peut être fait à partir de ces données montrant des résultats tout à fait similaires à ceux obtenus dans la première série de tests (figure 2).

\subsubsection{Changements granulométriques sur l'avant-côte de la Langue de Barbarie}

Les sédiments récoltés sur l'avant-côte de la Langue de Barbarie en juin et octobre 1990 à $10 \mathrm{~m}$ de profondeur sont des sables fins dont le mode granulométrique varie entre 2,92 et $3,43 \phi(0,133$ à $0,093 \mathrm{~mm}$; tableau $I I I)$. Le mode demeure pratiquement inchangé $(3,41 \phi$ en juin ct $3,47 \phi$ en octobre ; 0,094 à $0,09 \mathrm{~mm}$ ) mais les paramètres de triage, d'asymétrie et d'angulosité sont substantiellement différents. L'indice de triage est légèrement moins bon en octobre qu'en juin $(0,35$ au lieu de 0,22$)$; l'asy-
Tableau 1II. Données granulométriques du sable de l'avant-côte sénégalaise $(10 \mathrm{~m})$ utilisé dans le test de la figure 3 .

Table III. Grain-size data for nearshore sands sampled at $10 \mathrm{~m}$ off the north Senegal coast.

\begin{tabular}{ccc}
\hline $\begin{array}{c}\text { Diamètre } \\
(\mathbf{m m})\end{array}$ & juin 1990 & octobre $\mathbf{1 9 9 0}$ \\
\hline 2,000 & 0,0 & 0,0 \\
1,600 & & 0,2 \\
1,250 & 0,1 & 0,3 \\
1,000 & & 0,4 \\
0,730 & 0,2 & 0,6 \\
0,620 & 0,3 & 0,7 \\
0,500 & 0,4 & 0,8 \\
0,410 & 0,8 & 0,9 \\
0,290 & 0,9 & 1,1 \\
0,250 & 1,1 & 1,6 \\
0,210 & 2,0 & 3,1 \\
0,172 & 5,9 & 8,4 \\
0,125 & 12,2 & 20,4 \\
0,110 & 85,7 & 33,7 \\
0,080 & 93,9 & 93,5 \\
0,072 & 99,0 & 98,9 \\
0,050 & 100,0 & 99,9 \\
0,040 & & 100,0 \\
\hline
\end{tabular}

métrie devient très négative $(-0,42$ contre $-0,26)$, ce qui indique que la transformation texturale a entrâné une amélioration du triage du côté des fins ; l'angulosité enfin diminue $(1,28$ contre 1,57$)$, indication d'une courbe moins leptokurtique qui traduit un rééquilibrage du triage au profit des queues de distribution par rapport à la partie centrale. La simulation de la transformation globale qui rend compte du passage de la première structure granulométrique à l'assemblage observé en octobre a fait l'objet de plusieurs tests dont les caractéristiques sont indiquées dans le tableau IV. Les 12 tests furent réalisés selon la méthode des essais et des erreurs en sélectionnant différents groupements des trois paramètres directeurs $M, \sigma$ et $P$, de façon à modifier la courbe représentative du sédiment originel (celle de juin) pour qu'elle tende vers une distribution simulée suffisamment proche de la courbe finale d'octobre. Le test représente ici un processus 

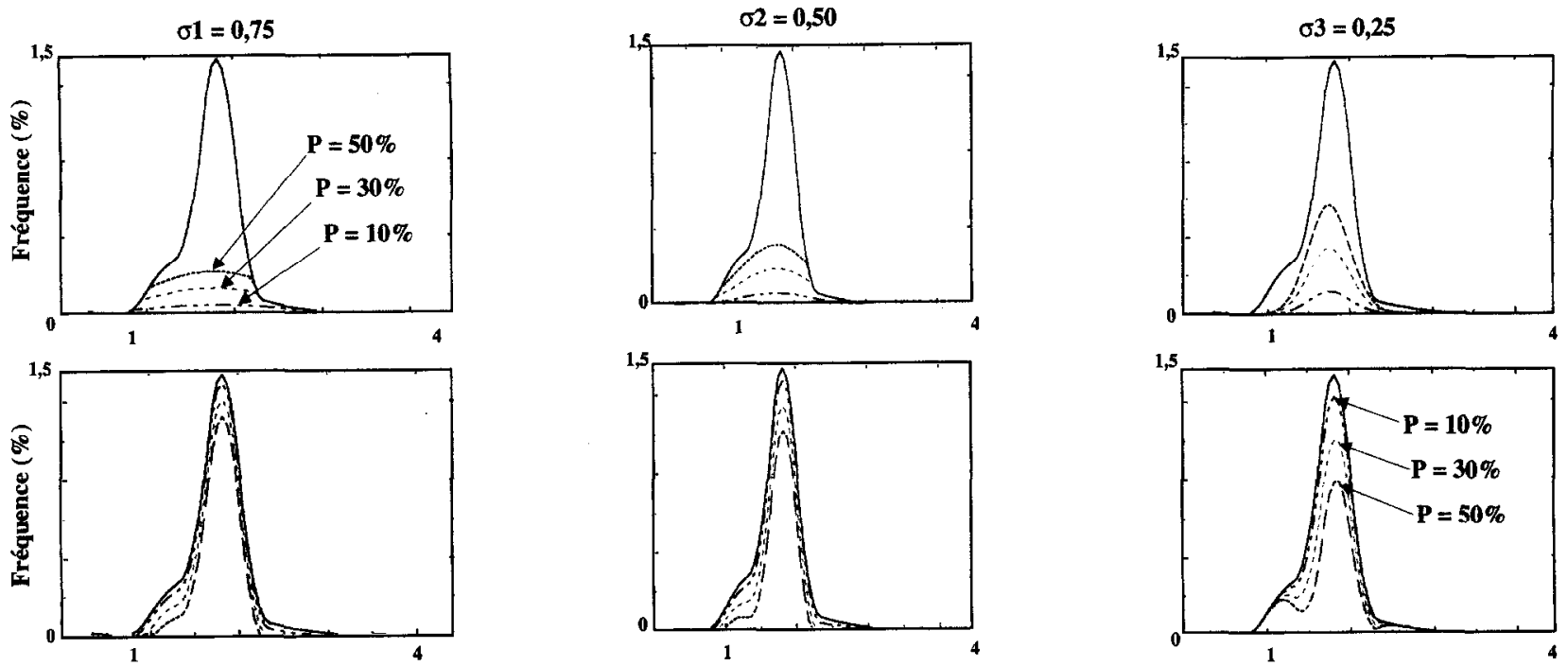

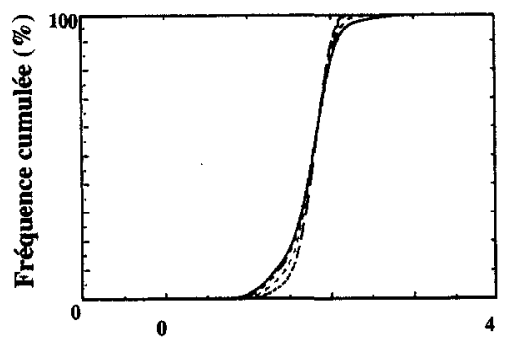

A

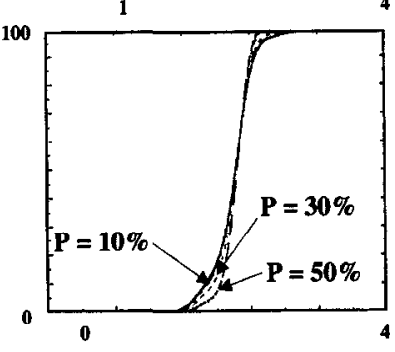

B

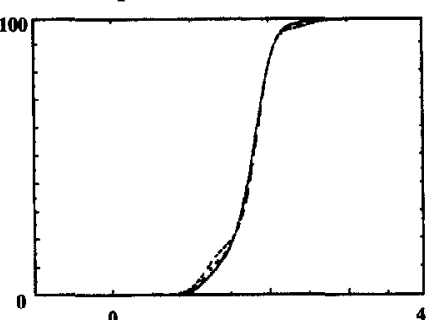

C

Figure 2. Simulation d'une extraction réalisée à partir d'une distribution réelle formalisée par une approximation de type cubic-spline. Chaque colonne se rapporte à une distribution d'écart type défini $(A: \sigma=0,75 \Phi ; B: \sigma=0,50 \Phi ; C: \sigma=0,25 \Phi)$. La courbe en trait gras représente la distribution initiale.

Figure 2. Automalic computation of extraction results operated on a natural grain-size curve approximated by cubic-spline fit. Each column is devoted to a particular standard deviation ( $\mathrm{A}: \sigma=0.75 \Phi ; \mathrm{B}: \sigma=0.50 \Phi ; \mathrm{C}: \sigma=0.25 \Phi$ ). Heavy line: grain-size curve of the initial sediment.

d'extraction sélective en raison de l'observation d'une érosion ayant accompagné la période de fortes houles d'hivernage.

Les meilleures solutions (en gras dans le tableau $I V$ ) sont obtenues quand la quantité de particules enlevées du sédiment est importante et centrée autour de la valeur modale.

Les résultats sont présentés dans la figure 3 où sont tracées les distributions initiale, dérivée après divers types d'extraction (selon les indications du tableau IV) et finale (celle-ci uniquement sur les deux schémas inférieurs). Les effets d'une modification portant à la fois sur l'écart type de la distribution du sédiment érodé et sur l'ampleur de cette érosion par rapport à la totalité du spectre granulométrique disponible permettent de connaître les caractères de la fraction érodée donnant la meilleure adé- quation avec le matériel dérivé. $\mathrm{Il}$ existe encore de notables disparités, mais un résultat approchant est obtenu quand, dans un intervalle limité $(\sigma=0,15 \phi$ donc $\mathrm{M} \pm 2 \sigma$ $=0,6 \phi)$, une fraction importante des grains disponibles dans cet intervalle $(75 \%)$ est enlevée (figure $3 a$ ). Le résultat est moins bon quand on opère une érosion avec une plus large dispersion (figure $3 b$ ). La faible dispersion de la fraction remaniée implique ici une grande sélectivité du facteur responsable de la transformation granulométrique.

\subsubsection{Changements granulométriques sur l'avant-côte du golfe du Lion}

Les sédiments récoltés dans la zone d'avant-côte en octobre 1994 sont des sables fins (tableau V). Après un coup 


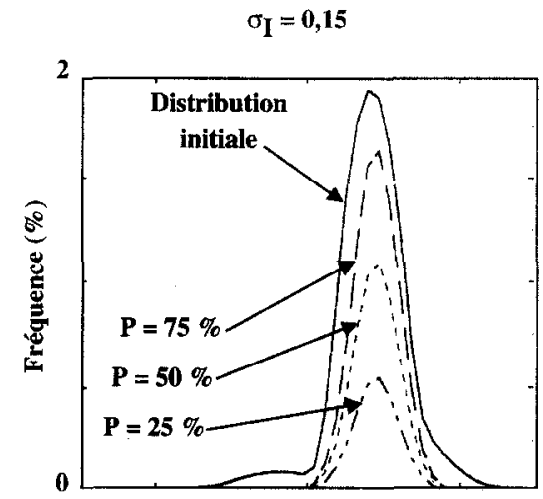

2 Dimension (unités $\phi$ ) 4

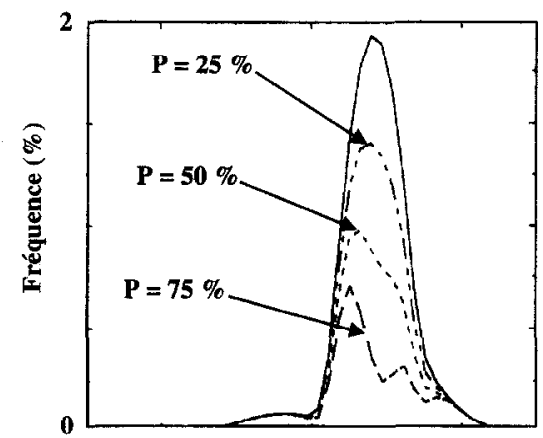

2

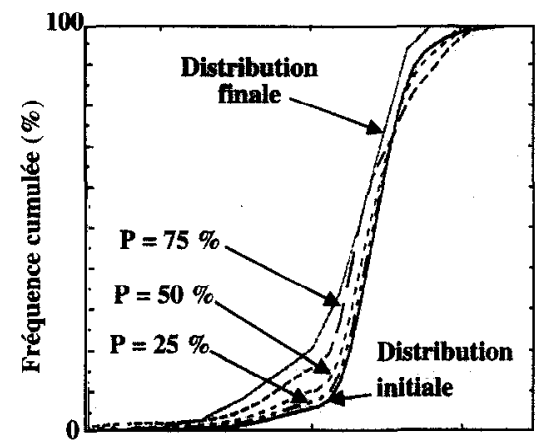

A

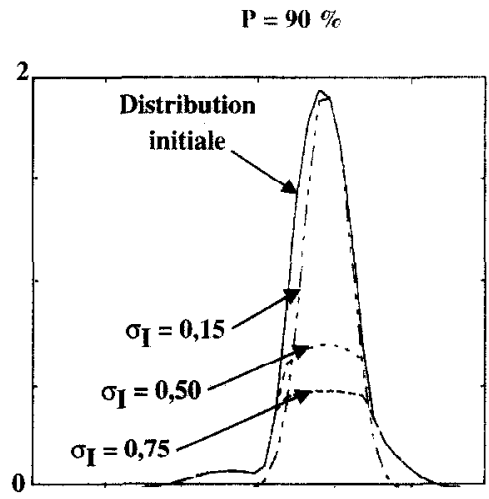

2

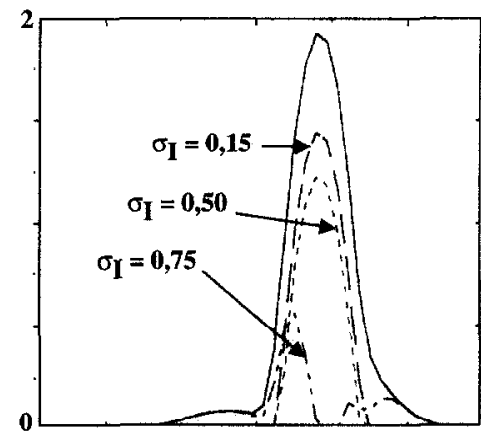

2

4

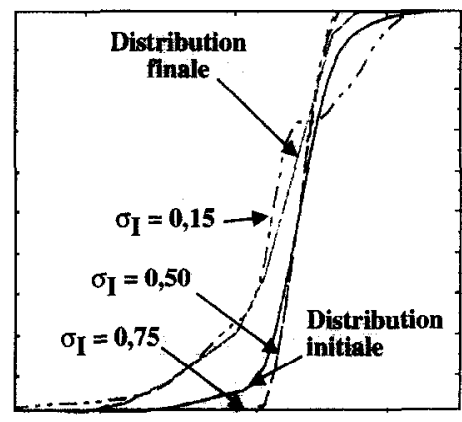

B

Figure 3. Simulation du passage entre une distribution réelle initiale (sédiment avant remaniement) et une distribution réelle finale (sédiment après remaniement), par essais et erreurs, illustrant les effets du paramètre de triage (colonne $\mathrm{A}$ ) et de l'ampleur $\mathrm{P}$ du prélèvement (colonne $\mathrm{B}$ ). Le sédiment provient des fonds de $10 \mathrm{~m}$ au large de la côte nord du Sénégal. Même légende que la figure $I$. Dans la colonne $\mathrm{A}(\sigma=0,15 \phi)$, le meilleur ajustement est obtenu pour une extraction représentant $75 \%$ du matériel disponible dans l'intervalle $\mathrm{M} \pm 2 \sigma$. Dans la colonne B $(\mathrm{P}=90 \%)$, le meilleur ajustement est obtenu pour un écart type étroit $(\sigma=0,15 \phi)$ mais la distribution finale simulée s'écarte de la distribution finale réelle du côté des fins.

Figure 3. Effects of sorting (A) and intensity (B) values of the extracted fraction on the conversion from an initial grain-size distribution into a final one (sediment at $10 \mathrm{~m}$ depth off the north Senegal coast).

(Same legend as figure 1 ; in column $\Lambda(\sigma=0.15 \phi)$, the best fit is obtained for an extraction corresponding to $75 \%$ of the material available in the interval $\mathrm{M} \pm 2 \sigma$; in column $\mathrm{B}(\mathrm{P}=90 \%)$, the best fit is obtained for a narrow standard deviation $(\sigma=0.15 \phi)$ ) but the distribution departs from the final one in the fine end.) 
Tableau IV. Tests combinant la variation de $M, \sigma$ et $P$ pour simuler la transition de la structure granulométrique du sable des fonds de $10 \mathrm{~m}$ (Sénégal) en juin 1990 vers celle du même sédiment en octobre 1990. Dans chaque test, les différents $\sigma$ sont combinés avec les différentes valeurs de $\mathrm{P}(\sigma=0,1 \phi$ est combiné successivement avec $\mathrm{P}=10,30$ et $50 \%$ etc. $)$.

Table IV. Various tests (combination of $\mathrm{M}, \sigma$ and $\mathrm{P}$ variations) illustrating the change from the June 1990 assemblage to the October 1990 structure. (In each test the various $\sigma$ were combined with the different values of P; i.c. test nb 1 was run 9 times with $\sigma=0.1 \phi$ combined successively with $P=10,30$ and $50 \%$ etc.)

\begin{tabular}{|c|c|c|c|c|}
\hline$n^{\circ}$ de test & M (unités $\phi$ ) & $\mathbf{M}(\mathbf{m m})$ & $\sigma$ (unités $\phi$ ) & $\mathbf{P}(\%)$ \\
\hline \multirow[t]{4}{*}{1} & 3,74 & 0,075 & 0,1 & 10 \\
\hline & & & 0,5 & 30 \\
\hline & & & 0,75 & 50 \\
\hline & & & & 75 \\
\hline \multirow{4}{*}{2} & 3,74 & 0,075 & 0,05 & 10 \\
\hline & & & 0,5 & 30 \\
\hline & & & 0,75 & 50 \\
\hline & & & & 75 \\
\hline \multirow[t]{4}{*}{3} & 3,74 & 0,075 & 0,05 & 10 \\
\hline & & & 0,5 & 30 \\
\hline & & & 0,75 & 50 \\
\hline & & & & 90 \\
\hline \multirow[t]{4}{*}{4} & 3,45 & 0,092 & 0,05 & 10 \\
\hline & & & 0,5 & 30 \\
\hline & & & 0,75 & 50 \\
\hline & & & & 90 \\
\hline \multirow[t]{4}{*}{5} & 3,55 & 3,55 & 0,2 & 25 \\
\hline & & & 0,5 & 50 \\
\hline & & & 0,75 & 75 \\
\hline & & & & 90 \\
\hline \multirow[t]{4}{*}{6} & 3,55 & 0,086 & 0,15 & 25 \\
\hline & & & & 50 \\
\hline & & & & 75 \\
\hline & & & & 90 \\
\hline \multirow[t]{4}{*}{7} & 3,45 & 0,092 & 0,2 & 25 \\
\hline & & & 0,5 & 50 \\
\hline & & & 0,75 & 75 \\
\hline & & & & 90 \\
\hline \multirow[t]{4}{*}{8} & 3,45 & 0,092 & 0,15 & 25 \\
\hline & & & & 50 \\
\hline & & & & 75 \\
\hline & & & & 90 \\
\hline \multirow[t]{4}{*}{9} & 3,45 & 0,092 & 0,13 & 75 \\
\hline & & & 0,15 & 80 \\
\hline & & & 0,16 & 85 \\
\hline & & & & 90 \\
\hline \multirow[t]{4}{*}{10} & 2,75 & 0,148 & 0,25 & 10 \\
\hline & & & 0,50 & 30 \\
\hline & & & 0,75 & 50 \\
\hline & & & & 90 \\
\hline \multirow[t]{3}{*}{11} & 3,17 & 0,11 & 0,25 & 10 \\
\hline & & & 0,50 & 30 \\
\hline & & & 0,75 & 50 \\
\hline \multirow[t]{3}{*}{12} & 3,74 & 0,075 & 0,25 & 10 \\
\hline & & & 0,50 & 30 \\
\hline & & & 0,75 & 50 \\
\hline
\end{tabular}

Tableau V. Données granulométriques du sable de l'avant-côte du golfe du Lion $(5 \mathrm{~m})$ utilisé dans le test de la figure 4.

Table V. Grain-size data for nearshore sands sampled at a depth of $5 \mathrm{~m}$ off the northwestern Mediterranean coast.

\begin{tabular}{lcc}
\hline $\begin{array}{c}\text { Diamètre } \\
(\mathbf{m m})\end{array}$ & $\begin{array}{c}\text { \% cum. } \\
\text { 18 octobre 1994 }\end{array}$ & $\begin{array}{c}\text { \% cum. } \\
\text { 21 octobre 1994 }\end{array}$ \\
\hline 3,15 & 100,00 & \\
2,5 & 99,20 & \\
2 & 98,40 & \\
1,6 & 98,20 & \\
1,25 & 98,10 & 100,00 \\
1 & 97,90 & 98,40 \\
0,8 & 97,70 & 98,20 \\
0,63 & 97,50 & 97,80 \\
0,5 & 97,20 & 97,40 \\
0,4 & 97,00 & 96,80 \\
0,315 & 96,30 & 94,90 \\
0,25 & 90,80 & 85,80 \\
0,2 & 58,00 & 52,90 \\
0,16 & 17,40 & 10,70 \\
0,125 & 3,30 & 0,90 \\
0,1 & 0,30 & 0,30 \\
0,08 & 0,20 & 0,20 \\
0,063 & & 0,10 \\
0,05 & & 0,00 \\
0,04 & 0,00 & \\
\hline
\end{tabular}

de mer modéré, la valeur $\phi$ du mode augmente car le sédiment s'affine $(2,39 \phi$ le 18 octobre, $2,60 \phi$ le 21 soit 0,19 à $0,165 \mathrm{~mm})$. Le triage s'améliore $(0,32$ avant, 0,23 après), l'asymétrie se dégrade après l'événement, devenant légèrement positive $\left(\mathrm{S}_{\mathrm{KI}}=0,21\right)$, ce qui indique un meilleur triage de la fraction grossière du sédiment. La partie centrale de la distribution voit son triage s'améliorer par rapport à celui des extrémités ( $\mathrm{K}_{\mathrm{G}}$ passant de 1,28 à 2,07). Les deux indices matérialisent une perturbation affectant principalement la fraction fine pendant le coup de mer.

La transformation qui rend le mieux compte du passage de la première structure granulométrique (18 octobre) à la distribution finale ( 21 octobre), est obtenue ici en ajoutant une fraction sableuse normalement distribuée dont les caractères sont illustrés sur la figure $4: \mathrm{M}=2,67 \phi$ $(0,157 \mathrm{~mm}) ; \sigma=0,32 \phi$ et $P=10-50 \%$.

\section{DISCUSSION}

Pour définir les caractères granulométriques des populations mobilisées lors d'une phase d'érosion-transport- 

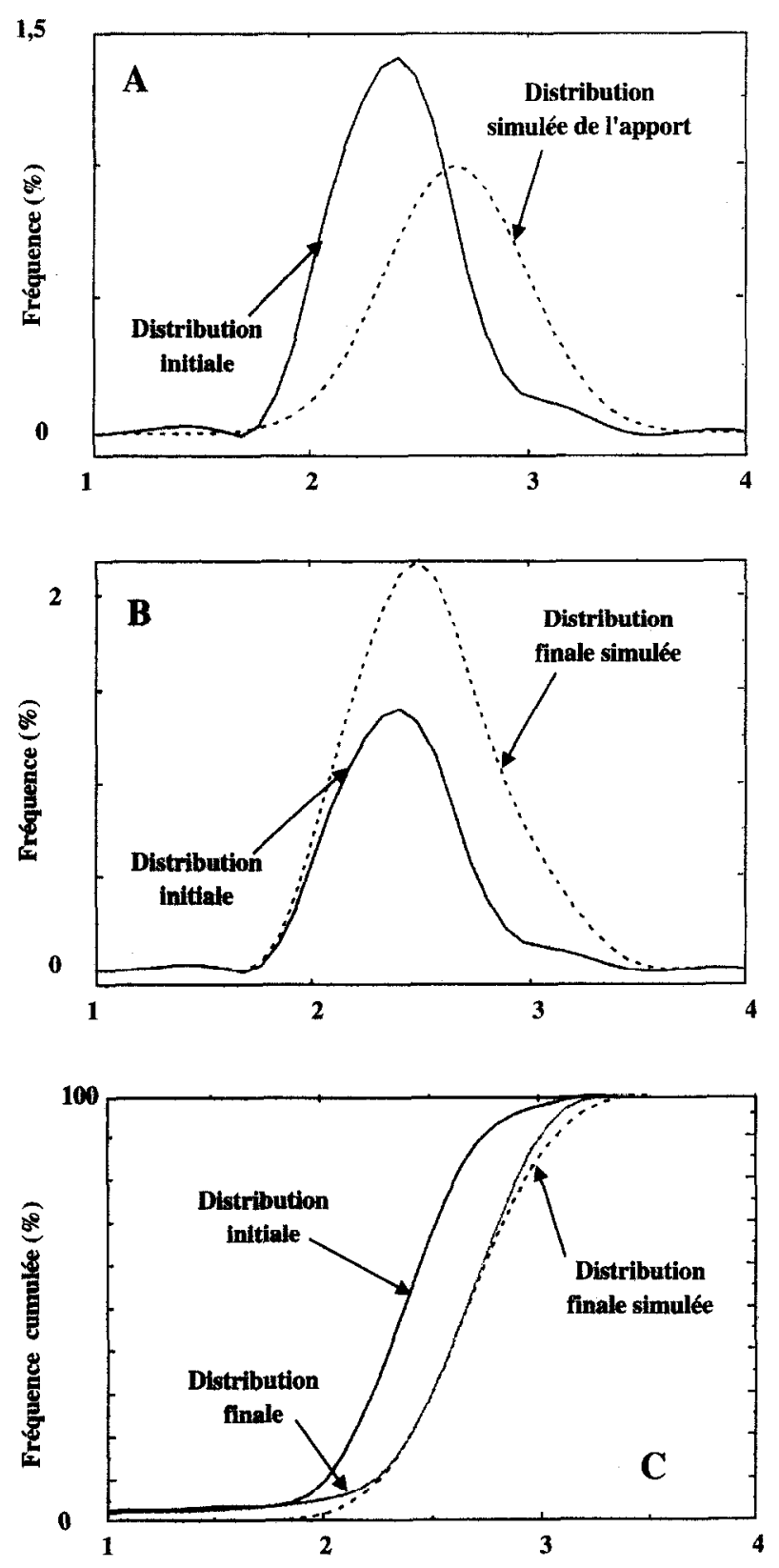

dépôt, on a comparé les états successifs de la texture des sédiments en un lieu donné. Les différentes questions successivement posées dans cette démarche furent: (1) qucllc représentation mathématique est-il possible de donner à une courbe granulométrique expérimentale ? (2) quelle fonction faut-il utiliser pour décrire de manière acceptable la fraction mobile? (3) les résultats des simulations faites en utilisant ces réponses sont-ils significatifs ?
Figure 4. Simulation du passage entre une distribution réelle initiale (sédiment avant apport extérieur) et une distribution réelle finale (sédiment après apport extérieur) par introduction d'une population gaussienne.

Le sédiment initial provient des fonds de $-5 \mathrm{~m}$ dans le golfe du Lion ; $M=2,67 \phi(0,157 \mathrm{~mm}) ; \sigma=0,32 \phi$. Même construction que pour les figures $1-3$; A : courbes de fréquence du sédiment prélevé le 18 octobre 1994, avant un coup de mer, et de l'apport présumé (après essais et erreurs selon le protocole développé dans l'étude) ; B : résultat de l'addition comparé à la courbe initiale (attention, le changement de hauteur de cette dernière par rapport à la figure $4 a$ est lić à un changement d'échelle d'unité de fréquence) ; $\mathrm{C}$ : comparaison de la distribution finale réelle (sédiment du 24 octobre 1994, au même endroit, après le coup de mer) avec la distribution finale simulée.

Figure 4. Change in the grain-size distribution of an offshore sand ( $-5 \mathrm{~m}$, Western Mediteranean) simulated by mixing a Gaussian distributed fraction to the initial sediment.

$(M=2.67 \phi=0.157 \mathrm{~mm} ; \sigma=0.32 \phi$; same construction as in figure 1 to 3; A: Frequency curves of the sediment sampled on 18 October 1994 and the simulated deposit; B: Initial and simulated grain-size distributions; C: Cumulative frequency curves of initial, final and simulated distributions).

\subsection{Expression de la fonction granulométrique et solution choisie}

Les distributions des sédiments clastiques sont difficilement exprimables par une seule fonction mathématique même si des approximations peuvent être parfois utilement employées. Les plus communes sont celles de Gauss [48], de Rosin [37] et la fonction log-hyperboli- 
que $[2,49]$. D'autres tentatives montrent que cette voie est loin d'être totalement explorée [46]. Les sédiments évolués suivent en général assez correctement une distribution gaussienne en raison de l'ajustement granulométrique qui s'opère entre les tailles des particules transportées et les agents hydrodynamiques responsables. La distribution tend alors à devenir symétrique même si un indice de triage $>1$ montre, le plus souvent, que les processus de sélection granulométrique sont inégalement actifs sur la totalité du spectre dimensionnel. Cependant l'ajustement d'une courbe de Gauss à une distribution granulométrique réelle est loin d'être complètement satisfaisant. Certains auteurs ont ainsi présumé que l'assemblage pouvait être un mélange de populations gaussiennes, symptomatiques des contributions respectives des modalités ordinaires du transport [50]. D'autres contestent aussi l'hypothèse de l'ajustement gaussien en considérant que le sédiment possède une asymétrie fondamentale résultant des conditions mêmes de la désagrégation originelle des roches dans les zones sources. Ils peuvent alors être représentés, selon eux, par une distribution, dite de Rosin, qui caractérisc des sédiments plutôt grossiers présentant une asymétrie positive et qui se rencontre aussi dans des sédiments résultant d'un processus rapide de dépôt [20]. Ce caractère pourrait être secondairement acquis comme l'ont montré Shih et Komar [42] décrivant le passage d'une distribution gaussienne à une distribution de Rosin par augmentation du débit et sélection résultante de grains arrachés au lit sédimentaire. Schleyer [37] applique ce concept à la définition d'un indice de maturité texturale ( Gauss and Rosin goodness-of-fit values »).

Une troisième catégorie d'auteurs considère que la recherche d'une équation représentative de la distribution est vaine. Ainsi, selon Kranck et Milliman [22], les processus de sédimentation ne sont pas liés à des phénomènes de hasard et ce type d'approximation ne peut convenir à la solution du problème posé. Cette option débouche sur un renoncement à toute formalisation et sur la préférence accordée au calcul d'indices par la méthode graphique ou la méthode des moments $[8,10,12,13,23$, 40]. Ces méthodes sont aussi critiquables [9]. Elles ne peuvent être appliquées qu'à des sédiments unimodaux et non à des matériaux polymodaux qui sont généralement des mélanges de populations simples $[3,4,16,31]$. Le cas des bimodalités qui apparaissent dans différents milieux, en particulier dans la zone littorale, complique de ce fait le problème de la formalisation mathématique des distributions naturelles car elles peuvent résulter de mélanges de populations [45] ou d'extractions sélectives de certaines fractions granulométriques [3].

La conséquence est que toute courbe réelle ne peut être modélisée par une fonction unique et ne peut être simulée qu'en faisant appel à des techniques d'approximation dont l'usage est facilité par l'emploi des ordinateurs de bureau. Ce constat a conduit au choix des méthodes cubic-spline qui fournissent des résultats satisfaisants et des temps de calcul assez courts sur les machines habituelles.

\subsection{Expression de la distribution granulométrique des fractions mobiles}

La seconde question abordée dans cette étude est la caractérisation des ensembles granulométriques mobilisés, soit par enlèvement, soit par apport de grains (fractions mobiles). Peu de données sont disponibles sur la distribution du matériel entraîné par un agent. Dans le domaine de la mobilité des sables sous l'influence du vent, plus accessible à l'expérience, Li et Martz [25] notent que l'influence de la texture sur le transport est considérable tandis que Rice et al. [34] soulignent l'impossibilité de définir la taille des grains éjectés par l'impact de grains de dimensions connues. Dans les milieux aquatiques, des résultats obtenus par Sengupta [39] indiquent que le matériel peut avoir une forme gaussienne, un résultat que retrouvent Bennett et Bridge ([5], figure 22). Sur cette base et malgré les réserves formulées plus haut sur la validité de la représentation gaussienne de la distribution des dépôts, nous avons estimé qu'une distribution de Gauss fournissait une approximation acceptable de la fraction en transport. Les simulations qui ont été faites ici pour transformer une courbe source réelle en sa courbe héritée (celle qui représente le même sédiment mais après l'action de processus hydrosédimentaires) ont respecté cette hypothèse. D'autres hypothèses devront être testées ultérieurement, en effet, Sengupta [39] a également rapporté une augmentation de l'asymétrie de la courbe granulométrique du matériel mis en suspension pour des vitesses faibles d'écoulement, voire le transport d'un stock granulométrique bimodal.

\subsection{Simulation d'enlèvement et de dépôt de grains (fraction mobile)}

La méthode de simulation utilisée montrc, dans deux cas de figures différents, qu'il est possible de fournir une assez bonne représentation de la fraction mobile. Elle est 
caractérisée : (1) par sa dimension modale (donc la valeur des grains les plus fréquemment déplacés), (2) par son écart type (caractérisant donc la plus grande partie du spectre mobilisé autour du mode) et (3) l'importance de la perturbation par rapport à ce qui cxistait auparavant dans le même intervalle.

Les résultats présentés sont tout à fait préliminaires mais il est certain que cette voie de recherche est prometteuse et doit permettre, en premicr licu, de micux définir la nature du matériel érodé, transporté puis déposé lors des processus hydrosédimentaires et, en second lieu, de mieux intégrer le paramètre granulométrique dans les modélisations numériques du transport par la prise en considération de la totalité du spectre dimensionnel.

\section{CONCLUSION}

La détermination par le calcul des flux sédimentaires sableux est pénalisée par une connaissancc insuffisante dc la texture des fractions mobiles. Leur détermination experimentale dans des pièges à particules est sujette à de grosses difficultés dans les zones où a lieu le transfert. Une méthode indirecte est décrite ici qui permet de reconnaître les paramètres directeurs de la structure granulométrique de la fraction, supposée d'allure gaussienne, qui exprime la mutation d'une distribution granulométrique d'un sédiment bien identifié dans une autre après un ou plusieurs événements hydrodynamiques. L'application de la méthode consiste en une série d'essais visant à obtenir le meilleur ajustement visuel entre la courbe expérimentale et la courbe simulée. La fraction mobile est alors définie par son mode granulométrique, son écart type et un facteur d'influence (proportion du matériel affecté par la transformation). On constate notamment que l'écart type de la fraction mobilisée au cours du changement granulométrique est le plus souvent relativement étroit $(0,1$ à 0,30 dans les deux exemples présentés). La méthode permet également de mettre en évidence l'enlèvement de particules ou au contraire leur dépôt.

\section{RÉFÉRENCES}

[1] Akouango E., Morphodynamique et dynamique sédimentaire dans le golfe du Lion, contribution à l'étude de la zone côtière dans l'actuel et le Quaternaire récent, thèse de doctorat non publiée, université de Perpignan, 1997, $189 \mathrm{p}$.

[2] Barndorff-Nielsen O.E., Blæsild P., Jensen J.L., Sørensen M., The fascination of sand, in: A celebration of statistics, Atkinson A.C., Fienberg S.E., (Eds.), Springer-Verlag, New York, (1985) 57-87.

[3] Barusseau J.P., Évolution du plateau continental rochelais (golfe de Gascogne) au cours du Pléistocène terminal et de l'Holocène. les processus actuels de la sédimentation, thèse non publiée, université de Bordeaux, 1973, 363 p. + ann.

[4] Bein A., Sass E., Analysis of log-probability plots of recent Atlantic sediments and its analogy with simulated mixtures, Sedimentology 25 (1978) 575-581.

[5] Bennett S.J., Bridge J.S., An experimental study of flow, bedload transport and bed topography under conditions of erosion and deposition and comparison with theoretical models, Scdimentology 42 (1995) 117-146.

[6] Chapalain G., Étude hydrodynamique et sédimentaire des environnements littoraux dominés par la houle, thèse non publiée, université Joseph-Fourier, Grenoble-I, 1988, 318 p.

[7] De Vriend H.J., G6/G8 coastal morphodynamics: introduction, Mast Days and Euromar Market, 15-17 March 1993, Part II: Coastal Morphodynamics and Structures, Project Reports 2 (1993) 441-463.
[8] Doeglas D.J., Grain-size indices, classification and environment, Sedimentology 10 (1968) 83-100.

[9] Ehrlich R., Size analysis wears no clothes, or have moments come and gone, J. Sedim. Petrol. editorial, 53, 1 (1983)

[10] Folk R.L., A review of grain-size parameters, Sedimentology, 6 (1966) 73-93.

[11] Folk R.L., Ward W.C., Brazos River bar: a study in the significance of grain-size parameters, J. Sedim. Petrol. 27 (1957) $3-26$.

[12] Friedman G.M., Dynamic processes and statistical parameters compared for size frequency distribution of beach and river sands, J. Sedim. Petrol. 37 (1967) 327-354.

[13] Friedman G.M., Address of the retiring President of the International Association of Sedimentologists : differences in size distributions of populations of particles among sands of various origins, Sedimentology 26 (1979) 3-32.

[14] Gao S., Collins M., A critique of the "McLaren method" for defining sediment transport paths, J. Sedim. Petrol. 61 (1991) 143-146.

[15] Gao S., Collins M., Analysis of grain-size trends for defining sediment transport pathways in marine environments, J. Coast. Res. 10 (1) (1994) 70-78.

[16] Goldberry R., Use of grain-size frequency data to interpret the depositional environment of the Pliocene Pleshet Formation, beer Sheva, Israël, J. Sedim. Petrol, 50 (1980) 843-856. 
[17] Greenwood B., Hale P.B., Lagrangian sediment motion in a crescentic bar under storm-induced waves and currents, J. Canad. Sci. Terre, 19, 3 (1982) 424-433.

[18] Haner B.E., Santa Ana River: an example of a sandy braided floodplain system showing sediment source area imprintation and selective sediment modification, Sedim. Geol, 38 (1984) 247-261

[19] Jago C.F., Barusseau J.P., Sediment entrainment on a wavegraded shelf, Roussillon, France, Mar. Geol. 42 (1981) 279, 299.

[20] Kittleman L.R., Application of Rosin's distribution in size frequency analysis of clastic rocks, J. Sedim. Petrol. 34 (1964) $483-502$.

[21] Kothyari U.C., Frequency distribution of river bed materials, Sedimentary 42 (2) (1995) 283-291.

[22] Kranck K., Milligan T.G., Origin of grain-size spectra of suspension deposited sediments, Geo-Mar. Lett. 5 (1985) 61-66.

[23] Krumbein W.C., Size-frequency distributions of sediments and the normal phi-curve, J. Sedim. Petrol. 8 (1938) 84-90.

[24] Levoy F., Évolution et fonctionnement hydrosédimentaire des plages macrotidales, l'exemple de la côte ouest du Cotentin, thèse non publiée, université de Caen Basse-Normandie, 1994, 423 p. + ann.

[25] Li L., Martz L.W., Aerodynamic dislodgement of multiplesize sand grains over time, Sedimentology 42 (1995) 683-694.

[26] Liu H.K., Mechanics of ripple formation, J. Hydraul. Division, ASCE, 83 HY2, (1957) 1197.

[27] McCave I.N., Grain-size trends and transport along beaches: examples from eastern England, Mar. Geol. 28 (1978) M43M51.

[28] McLaren P., An interpretation of trends in grain size measures, J. Sedim. Petrol. 51 (1981) 611-642.

[29] McLaren P., Bowles D., The effects of sediment transport on grain-size distributions, J. Sediment. Petrol. 55 (1985) 454470.

[30] Michel P., Barusseau J.P., Richard J.F., Sall M., L'après barrages dans la vallée du Sénégal, modifications hydrodynamiques et sédimentologiques, coll. études, Presses Universitaires de Perpignan (1993) $152 \mathrm{p}$.

[31] Michels K.H., The decomposition of polymodal settling velocity distributions for a comprehensive sedimentological description of sand-sized samples, Sedimentology 42 (1995) 31-38.

[32] Moss A.J., The physical nature of common sandy pebbly deposits, part I, Amer. J. Sci. 260 (1962) 337-373.

[33] Pedreros R., Howa H.L., Michel D., Application of grain size trend analysis for the determination of sediment transport pathways in intertidal areas, Mar. Geol. 135 (1996) 35-49.
[34] Rice M.A., Willetts B.B., McEwan I.K., An experimental study of multiple grain-size ejecta produced by collisions of saltating grains with a flat bed, Sedimentology 42 (1995) 695 706 .

[35] Roelvink J.A., Brøker I., Cross-shore profile models, Coast. Engin. 21 (1993) 163-191.

[36] Sarre R.D., Chancey C.C., Size segregation during aeolian saltation on sand dunes, Sedimentology 37, (1990) 357-365.

[37] Schleyer R., The goodness-of-fit to ideal Gauss and Rosin distributions: a ncw grain-size parameter, J. Sediment. Petrol. 57 (1987) 871-880.

[38] Sengupta S., Size-sorting during suspension transportation Lognormality and other characteristics, Sedimentology 22 (1975) 257-273.

[39] Sengupta S., Grain-size distribution of suspended load in relation to bed materials and flow velocity, Sedimentology 26 (1979) 6382.

[40] Seward-Thompson B.L., Hails J.R., An appraisal of the computation of statistical parameters in grain size analysis. Sedimentology 20 (1973) 161-169.

[41] Shih S.M., Komar P.D., Differential bedload transport rates in a gravel-bed stream: a grain-size distribution approach, Earth Surface Processes and Landforms, 15 (1990a) 539-552.

[42] Shih S.M., Komar P.D., Hydraulic controls of grain-size distributions of bedload gravels in Oak Creek, Oregon, USA, Sedimentology 37 (1990b) 377-384.

[43] Singer J.K., Anderson J.B., Use of total grain-size distributions to define bed erosion and transport for poorly sorted sediment undergoing simulated bioturbation, Mar. Geol. 59 (1984) 335-359.

[44] Stapor F.W., Tanner W.F., Hydrodynamic implication of beach, beach ridge and dune grain-size studies, J. Sediment. Petrol. 45 (1975) 926-931.

[45] Taira A., Scholle P.A., Origin of bimodal sands in some modern environments, J. Sediment. Petrol. 49 (1979) 777-786.

[46] Tauber F., Lemke W., Map of sediment distribution in the Western Baltic Sea $1: 100,000$., sheet “Darß", Deutsche Hydrographische Zeitschrift 47 (3) (1995) 171-178.

[47] Thibault M., Barusseau J.P., Deux exemples des modalités de triage granulométrique sur le littoral et l'avant-côte, Proceedings of the IXth International Congress of Sedimentology, Nice, 6 (1975) 205-210.

[48] Udden J.A., Mechanical composition of clastic sediments, Geol. Soc. Amer. Bull. 25 (1914) 655-744.

[49] Vincent P., Differenciation of modern beach and coastal dune sands, A logistic approach using the parameters of the hyperbolic function, Sedim. Geol. 49 (1986) 167-176.

[50] Visher G.S., Grain-size distributions and depositional processes, J. Sedim. Petrol. 39 (1969) 1074-1 106. 